01

\title{
Ядерное магнитное экранирование в многозарядных ионах
}

\author{
(ㄱ А.М. Волчкова, Д.А. Глазов, В.М. Шабаев \\ Санкт-Петербургский государственный университет, \\ 199034 Санкт-Петербург, Россия \\ e-mail: glazov.d.a@gmail.com
}

Поступила в редакцию 27.08.2021 г.

В окончательной редакции 27.08.2021 г.

Принята к публикации 10.09.2021 г.

\begin{abstract}
Эффект ядерного магнитного экранирования рассмотрен в рамках полностью релятивистского подхода для основного состояния H-, Li- и В-подобных ионов в диапазоне $Z=32-92$. Межэлектронное взаимодействие вычислено в первом порядке теории возмущений в Li- и В-подобных ионах. Расчеты основаны на методе конечного поля. Применяется численное решение уравнения Дирака с учетом магнитного и сверхтонкого взаимодействий в рамках метода дуального кинетического баланса. Константа ядерного магнитного экранирования является важной составляющей для определения ядерных магнитных моментов из высокоточных измерений $g$-фактора.
\end{abstract}

Ключевые слова: многозарядные ионы, g-фактор, сверхтонкая структура, ядерное магнитное экранирование.

DOI: $10.21883 /$ OS.2021.12.51733.2682-21

\section{1. Введение}

Многозарядные ионы являются универсальным инструментом для проверки Стандартной модели, поиска новой физики, определения фундаментальных констант и ядерных параметров [1-4]. В частности, ядерные магнитные моменты находятся в центре внимания в настоящее время, поскольку погрешность их определения из наиболее распространённого метода ядерного магнитного резонанса может оказаться значительно больше, чем принято считать $[5,6]$. Независимый метод, свободный от эффектов окружающей среды, которые трудно оценить, был предложен в [7] и развит в [8]. Он основан на измерении $g$-фактора в многозарядных ионах, где за последние два десятилетия был достигнут поразительный прогресс [9]. Относительная экспериментальная погрешность достигла $2.4 \cdot 10^{-11}$ в Н-подобном углероде [10], $0.7 \cdot 10^{-10}$ в Li-подобном кремнии $[11]$ и $1.4 \cdot 10^{-9}$ в Bподобном аргоне [12]. Определение ядерного магнитного момента, в дополнение к прецизионным измерениям, требует точных теоретических предсказаний в рамках квантовой электродинамики (КЭД) для связанных состояний. Одновременно с экспериментом релятивистская теория $g$-фактора связанного электрона получила значительное развитие в последние годы $[13,14]$. В ионах с ненулевым ядерным спином возникает поправка к $g$-фактору на сверхтонкое взаимодействие $\delta g_{\text {HFs. }}$ Она также может быть выражена в терминах константы ядерного магнитного экранирования $\sigma$. Этот вклад был подробно исследован в рамках КЭД-теории для связанных состояний в Н-подобных [15-17] и Li-подобных [18] ионах. Нелинейные эффекты в магнитном поле, описываемые формулой Брейта-Раби, рассматривались, в частности, в работах $[19,20]$.
В указанных выше работах как сверхтонкое взаимодействие, так и взаимодействие с внешним магнитным полем рассматривались в рамках теории возмущений. В качестве альтернативного подхода в работе [21] использовался так называемый метод конечного поля, основанный на решении уравнения Дирака, которое включает магнитное взаимодействие. Ведущий вклад в $\sigma$ был вычислен для состояний $1 s, 2 s$ и $2 p_{1 / 2}$, было обнаружено отличное согласие с теорией возмущений. Для основного состояния В-подобных ионов также были рассмотрены три различных эффективных экранирующих потенциала. На основании большого вклада экранировки был сделан вывод о необходимости более строгого рассмотрения межэлектронного взаимодействия. Это побудило нас к дальнейшему исследованию многоэлектронных эффектов в этих системах. В данной работе мы представляем расчеты поправки первого порядка по межэлектронному взаимодействию, так называемый однофотонный обмен, для $\mathrm{Li}$ - и В-подобных ионов в рамках метода конечного поля. Ранее эта поправка уже была получена для Li-подобных ионов в рамках теории возмущений [18]. Сравнение наших результатов с результатами из работы [18] демонстрирует правильность численной процедуры. Для В-подобных ионов данный вклад вычисляется впервые.

В работе используются релятивистские единицы $\left(\hbar=1, c=1, m_{e}=1\right)$ и единица заряда Хевисайда $\left(\alpha=e^{2} /(4 \pi), e<0\right), Z-$ заряд ядра, $\mu_{\mathrm{B}}=|e| / 2 m_{\mathrm{e}} c-$ магнетон Бора, $\mu_{\mathrm{N}}=|e| / 2 m_{\mathrm{p}} c-$ ядерный магнетон, $m_{\mathrm{e}}$ и $m_{\mathrm{p}}$ - массы электрона и протона соответственно.

\section{2. Теория}

Мы рассматриваем ион с одним электроном поверх замкнутых оболочек и ненулевым ядерным спином $I$. 
Уровни энергии с электронным угловым моментом $J$ расщепляются в зависимости от величины полного углового момента системы, $\mathbf{F}=\mathbf{J}+\mathbf{I}$. Релятивистскую теорию сверхтонкого расщепления и наиболее точные теоретические значения для $\mathrm{H}-, \mathrm{Li}-$ и В-подобных многозарядных ионов можно найти, например, в работах [2225]. В присутствии внешнего магнитного поля $\mathbf{B}$, направленного вдоль оси $z$, уровень энергии основного состояния иона расщепляется в соответствии с $z$-проекцией $M_{F}$ полного углового момента $F$. В предположении, что зеемановское расщепление намного меныше сверхтонкого, первое можно записать как

$$
\Delta E_{\mathrm{mag}}=g_{F} \mu_{\mathrm{B}} B M_{F} .
$$

Полный $g$-фактор системы даётся следующей формулой:

$$
g_{F}=g_{J} \frac{\langle\mathbf{J} \cdot \mathbf{F}\rangle}{\left\langle F^{2}\right\rangle}-\frac{m_{\mathrm{e}}}{m_{\mathrm{p}}} g_{I} \frac{\langle\mathbf{I} \cdot \mathbf{F}\rangle}{\left\langle F^{2}\right\rangle}+\delta g_{\mathrm{HFS}},
$$

где

$$
\begin{gathered}
\langle\mathbf{J} \cdot \mathbf{F}\rangle=\frac{1}{2}[F(F+1)+J(J+1)-I(I+1)], \\
\langle\mathbf{I} \cdot \mathbf{F}\rangle=\frac{1}{2}[F(F+1)+I(I+1)-J(J+1)], \\
\left\langle F^{2}\right\rangle=F(F+1) .
\end{gathered}
$$

Здесь $g_{J}$ - электронный $g$-фактор, $g_{I}$ - ядерный $g$ фактор, связанный с ядерным магнитным моментом как $\mu^{(n)}=g_{I} \mu_{\mathrm{N}} I$. Выражение (2) также может быть записано в виде

$$
g_{F}=g_{J} \frac{\langle\mathbf{J} \cdot \mathbf{F}\rangle}{\left\langle F^{2}\right\rangle}-(1-\sigma) \frac{m_{\mathrm{e}}}{m_{\mathrm{p}}} g_{I} \frac{\langle\mathbf{I} \cdot \mathbf{F}\rangle}{\left\langle F^{2}\right\rangle},
$$

где $\sigma$ называется константой ядерного магнитного экранирования. Связь между поправкой на сверхтонкое взаимодействие $\delta g_{\text {HFS }}$ и $\sigma$ выглядит следующим образом:

$$
\delta g_{\mathrm{HFS}}=\sigma \frac{m_{\mathrm{e}}}{m_{\mathrm{p}}} g_{I} \frac{\langle\mathbf{I} \cdot \mathbf{F}\rangle}{\left\langle F^{2}\right\rangle} .
$$

Для рассматриваемых ионов с одним электроном поверх замкнутых оболочек угловой момент $\mathbf{J}$ полностью определяется валентным электроном. В этом случае ведущий вклад в $\sigma$ (если пренебречь межэлектронным взаимодействием) может быть записан как

$$
\sigma_{0}=\alpha \sum_{n} \frac{\langle a|U| n\rangle\langle n|W| a\rangle}{\epsilon_{a}-\epsilon_{n}},
$$

где операторы

$$
U=[\mathbf{r} \times \boldsymbol{\alpha}]_{z}, \quad W=\frac{[\mathbf{r} \times \boldsymbol{\alpha}]_{z}}{r^{3}}
$$

возникают из операторов магнитного и сверхтонкого взаимодействий соответственно [21]. Состояние валентного электрона $|a\rangle$, промежуточные состояния $|n\rangle$ и их энергии $\epsilon_{a}$ и $\epsilon_{n}$ определяются из уравнения Дирака

$$
\left(\boldsymbol{\alpha} \cdot \mathbf{p}+\beta+V_{\mathrm{nuc}}(r)\right) \psi(\mathbf{r})=\epsilon \psi(\mathbf{r}) .
$$

Здесь $\alpha$ и $\beta-$ матрицы Дирака, $V_{\text {nuc }}(r)-$ потенциал ядра. Суммирование по спектру в уравнении (5) может быть выполнено с помощью метода конечного базисного набора, как это было сделано для Н-подобных [15] и Li-подобных [18] ионов. Для точечного ядра $\sigma_{0}$ также может быть получена аналитически [15,26-29].

Кроме того, $\sigma$ может быть найдена так называемым методом конечного поля, т.е. из энергий и волновых функций, которые учитывают магнитное и/или сверхтонкое взаимодействие. В работе [21] в уравнение Дирака было включено только магнитное взаимодействие $\lambda U$,

$$
\left(\boldsymbol{\alpha} \cdot \mathbf{p}+\beta+V_{\mathrm{nuc}}(r)+\lambda U\right) \psi(\mathbf{r}, \lambda)=\epsilon(\lambda) \psi(\mathbf{r}, \lambda),
$$

а $\sigma$ была получена как производная матричного элемента $\langle a(\lambda)|W| a(\lambda)\rangle$ относительно $\lambda$,

$$
\sigma_{0}=\left.\frac{\alpha}{2} \frac{\partial\langle a(\lambda)|W| a(\lambda)\rangle}{\partial \lambda}\right|_{\lambda=0} .
$$

В данной работе в уравнение Дирака включается как магнитное, так и сверхтонкое взаимодействие,

$$
\begin{aligned}
& \left(\boldsymbol{\alpha} \cdot \mathbf{p}+\beta+V_{\mathrm{nuc}}(r)+\lambda U+\mu W\right) \psi(\mathbf{r}, \lambda, \mu) \\
& =\epsilon(\lambda, \mu) \psi(\mathbf{r}, \lambda, \mu) .
\end{aligned}
$$

Затем $\sigma_{0}$ вычисляется путем взятия смешанной производной,

$$
\sigma_{0}=\left.\frac{\alpha}{2} \frac{\partial^{2} \epsilon(\lambda, \mu)}{\partial \lambda \partial \mu}\right|_{\lambda=0, \mu=0} .
$$

В более высоких порядках теории возмущений данный подход оказывается эффективнее, так как позволяет снять суммирование по спектру за счет повторения вычислений для набора точек по $\mu$ (см. ниже обсуждение поправки на однофотонный обмен).

В системах с несколькими электронами необходимо также учитывать взаимодействие между ними. В этой работе мы рассматриваем первый порядок теории возмущений, который представлен диаграммами однофотонного обмена. Этот вклад в $\sigma$ был вычислен в работе [18] для Li-подобных ионов, где можно найти соответствующие диаграммы и формулы. В данной работе мы рассматриваем его в рамках метода конечного поля, т. е. на основе решений уравнения (10). Поправка на однофотонный обмен к энергии связи валентного электрона выражается следующим образом:

$$
\Delta E_{1 \mathrm{ph}}=\sum_{b}\left(\langle a b|I(0)| a b\rangle-\left\langle b a\left|I\left(\epsilon_{\mathrm{a}}-\epsilon_{\mathrm{b}}\right)\right| a b\right\rangle\right) .
$$

Здесь $|a\rangle$ - состояние валентного электрона, а суммирование идет по состояниям замкнутых оболочек $|b\rangle$, оператор межэлектронного взаимодействия $I$ в калибровке Фейнмана записывается как

$$
I\left(\omega, \mathbf{r}_{12}\right)=\alpha\left(1-\boldsymbol{\alpha}_{1} \cdot \boldsymbol{\alpha}_{2}\right) \frac{\exp \left(i|\omega| r_{12}\right)}{r_{12}}
$$


Вклад однофотонного обмена в $\sigma$ можно найти из соответствующей поправки к энергии (12), взяв смешанную производную,

$$
\Delta \sigma_{1 \mathrm{ph}}=\left.\frac{\alpha}{2} \frac{\partial^{2} \Delta E_{1 \mathrm{ph}}(\lambda, \mu)}{\partial \lambda \partial \mu}\right|_{\lambda=0, \mu=0} .
$$

Здесь предполагается, что $\Delta E_{1 \mathrm{ph}}$ вычисляется по формуле (12) с волновыми функциями $|a\rangle$ и $|b\rangle$ и энергиями $\epsilon_{a}$ и $\epsilon_{b}$, которые удовлетворяют уравнению Дирака (10). Отметим, что это выражение не содержит сумм по спектру, которые обычно присутствуют во втором и более высоких порядках теории возмущений. В частности, выражение для $\Delta \sigma_{1 \mathrm{ph}}$ содержит двойное суммирование в рамках стандартной теории возмущений [18]. В рамках метода конечного поля с одной производной по $\lambda$ в соответствии с уравнениями (8) и (9) возникает однократное суммирование.

Чтобы реализовать предложенный способ нахождения $\sigma_{0}$ и $\Delta \sigma_{1 \mathrm{ph}}$, нам необходимо решить уравнение Дирака (10), которое обладает аксиальной, но не сферической симметрией. Эффективный численный подход для решения уравнения Дирака с аксиально симметричным потенциалом был предложен в работе [30]. Он обобщает метод дуального кинетического баланса [31] с базисными функциями, построенными из В-сплайнов [32]. Первоначально разработанный для исследования динамики электронов во внешнем лазерном поле он был применен также к таким аксиально симметричным задачам, как зеемановское расщепление в многозарядных ионах $[21,33,34]$ образование электрон-позитронных пар в столкновениях тяжелых ионов [35] и энергии связи в двухатомных молекулярных ионах [36-38]. Ниже мы кратко напомним основы этого метода.

В аксиально симметричном потенциале зависимость дираковской волновой функции от $\varphi$ может быть записана в явном виде,

$$
\psi(\mathbf{r})=\frac{1}{r}\left(\begin{array}{c}
G_{1}(r, \theta) \exp \left[i\left(M_{J}-\frac{1}{2}\right) \varphi\right] \\
G_{2}(r, \theta) \exp \left[i\left(M_{J}+\frac{1}{2}\right) \varphi\right] \\
i F_{1}(r, \theta) \exp \left[i\left(M_{J}-\frac{1}{2}\right) \varphi\right] \\
i F_{2}(r, \theta) \exp \left[i\left(M_{J}+\frac{1}{2}\right) \varphi\right]
\end{array}\right),
$$

где $M_{J}-z$-проекция углового момента $J$. Уравнение (10) сводится к $H_{M_{J}} \Phi=\epsilon \Phi$, где

$$
\begin{gathered}
H_{M_{J}}=\left(\begin{array}{cc}
1 & D_{M_{J}}+\tilde{D} \\
-\left(D_{M_{J}}+\tilde{D}\right) & -1
\end{array}\right), \\
D_{M_{J}}=\left(\sigma_{z} \cos \theta+\sigma_{x} \sin \theta\right)\left(\frac{\partial}{\partial r}-\frac{1}{r}\right)+\frac{1}{r} \\
\times\left(\sigma_{x} \cos \theta-\sigma_{z} \sin \theta\right) \frac{\partial}{\partial \theta}+\frac{1}{r \sin \theta}\left(i M_{J} \sigma_{y}+\frac{1}{2} \sigma_{x}\right), \\
\tilde{D}=\left(\lambda r+\frac{\mu}{r^{2}}\right) i \sigma_{y} \sin \theta .
\end{gathered}
$$

Член $\tilde{D}$ возникает из $\lambda U$ и $\mu W$, проинтегрированных по $\varphi$ с экспонентами из $\psi(\mathbf{r})(15), \sigma_{x, y, z}$ - матрицы Паули, а $\Phi-4$-компонентная волновая функция, которая зависит от $r$ и $\theta$,

$$
\Phi(r, \theta)=\left(\begin{array}{c}
G_{1}(r, \theta) \\
G_{2}(r, \theta) \\
F_{1}(r, \theta) \\
F_{2}(r, \theta)
\end{array}\right) .
$$

Функции $G_{k}(r, \theta)$ и $F_{k}(r, \theta)$ раскладываются по конечному базисному набору, построенному из В-сплайнов по переменной $r$ и полиномов по переменной $\theta / \pi$. Для устранения ложных состояний на этот базисный набор накладываются специальные условия дуального кинетического баланса, подробнее см. в работах $[30,31]$. Чтобы найти коэффициенты этого разложения и соответствующие энергии, численно решается обобщенная задача на собственные значения. Низшие состояния с положительной энергией соответствуют связанным состояниям уравнения (10). Все множество решений образует квазиполный конечный базисный набор, который эффективно воспроизводит бесконечный спектр, включая положительно- и отрицательно-энергетические континуумы, и может быть использован, в частности, для построения функции Грина уравнения (10).

Для вычисления $\sigma_{0}$ и $\Delta \sigma_{1 \mathrm{ph}}$ по формулам (11) и (14) необходимо найти энергии $\epsilon_{a, b}(\lambda, \mu)$ и волновые функции $|a\rangle,|b\rangle$ на множестве точек $\lambda=-n \lambda_{0}, \ldots,-\lambda_{0}, 0, \lambda_{0}, \ldots, n \lambda_{0} \quad$ и $\mu=-n \mu_{0}, \ldots,-\mu_{0}, 0, \mu_{0}, \ldots, n \mu_{0} \quad$ и $\quad$ использовать стандартные формулы для производных. Правильный выбор $\lambda_{0}$ и $\mu_{0}$ очень важен для получения точных результатов. Для выбора оптимальных значений $\lambda_{0}, \mu_{0}$ и $n$ проводится серия расчетов для различных значений этих параметров. Для расчетов, представленных в данной работе, мы используем $\lambda_{0}$ и $\mu_{0}$ в диапазоне $10^{-4} \ldots 10^{-7}$ и $n=1,2,3$.

\section{3. Результаты и обсуждение}

В этом разделе мы представляем результаты для константы ядерного магнитного экранирования в диапазоне $Z=32-92$, включая поправку на однофотонный обмен, полученные методом конечного поля, подробно описанным выше. При наличии ранее опубликованных значений мы приводим их для сравнения и во всех случаях отмечаем хорошее согласие. Для того чтобы дополнительно проверить представленный метод, мы также вычислили ведущий порядок сверхтонкого расщепления и соответствующую поправку на однофотонный обмен. Эти результаты представлены в терминах коэффициентов $A$ и $B / Z$ [22-25]. В этих работах $A$ вычислено для точечного ядра, а поправка на конечный размер ядра представлена множителем $1-\delta$. Мы проводим все расчеты для конечного ядра, поэтому обозначаем 
Таблица 1. Коэффициент сверхтонкого расщепления $A_{\text {fns }}$ и константа ядерного магнитного экранирования $\sigma_{0}$ для основного состояния $1 s$ Н-подобных ионов. Для $A_{\mathrm{fns}}$ приведены для сравнения результаты наших расчетов по теории возмущений и данные из работы [22]. Также представлены значения $\sigma_{0}=\alpha^{2} Z S(\alpha Z) / 3$ из работы [15]. Значения $\sigma$ приведены в единицах $10^{-3}$

\begin{tabular}{l|l|l}
\hline$Z$ & $A_{\text {fns }}$ & $\sigma_{0} \cdot 10^{3}$ \\
\hline 32 & 1.0813 & 0.6578 \\
& $1.0813^{a}$ & $0.6579^{b}$ \\
\hline 54 & 1.2687 & 1.4615 \\
& $1.2686^{a}$ & $1.4617^{b}$ \\
\hline 82 & 1.8537 & 3.9548 \\
& $1.8548^{a}$ & $3.9579^{b}$ \\
& $1.8545^{c}$ & \\
\hline 83 & 1.8881 & 4.1093 \\
& $1.8892^{a}$ & $4.1109^{b}$ \\
\hline 90 & $1.8889^{c}$ & \\
\hline 92 & 2.1688 & 5.397 \\
& $2.1694^{a}$ & 5.843 \\
& 2.2634 & $5.851^{b}$
\end{tabular}

Примечание. ${ }^{a}$ Теория возмущений, ${ }^{b}$ Moskovkin et al. [15], ${ }^{c}$ Shabaev et al. [22].

нашу величину как $A_{\text {fns }}$ и сравниваем ее с $A(\alpha Z)(1-\delta)$ (поправка Бора-Вайскопфа не учитывается). Значения $B$ из работ [23-25] учитывают эффект конечного размера ядра. Используя решения уравнения Дирака (10), эти коэффициенты могут быть найдены следующим образом:

$$
\begin{gathered}
A_{\mathrm{fns}}=\left.G_{a} \frac{\partial \epsilon(\lambda, \mu)}{\partial \mu}\right|_{\lambda=0, \mu=0}, \\
B / Z=\left.G_{a} \frac{\partial \Delta E_{1 \mathrm{ph}}(\lambda, \mu)}{\partial \mu}\right|_{\lambda=0, \mu=0}, \\
G_{a}=\frac{n^{3}(2 l+1) j(j+1)}{2(\alpha Z)^{3} M_{J}},
\end{gathered}
$$

где $n, l$ и $j$ - квантовые числа валентного электрона. Кроме того, мы вычисляем $A_{\mathrm{fns}}$ и $B / Z$ по теории возмущений, используя конечный базисный набор для уравнения (7), построенный в рамках метода дуального кинетического баланса [31].

Ядерное магнитное экранирование для основного состояния $1 s$ Н-подобных ионов было вычислено по теории возмущений, в частности работах [15-17]. В табл. 1 мы сравниваем результаты, полученные в данной работе методом конечного поля, и результаты, полученные в статье [15]. Значения константы $\sigma$ представлены в единицах $10^{-3}$. Значения функции $S(\alpha Z)$ из работы [15] умножаются на коэффициент $\alpha^{2} Z / 3$ для получения $\sigma$. Кроме того, мы сравниваем результаты для коэффициента сверхтонкого расщепления $A_{\text {fns }}$ с нашими расчетами
Таблица 2. Коэффициенты сверхтонкого расщепления ( $A_{\mathrm{fns}}$ и $B / Z)$ и константа ядерного магнитного экранирования $\left(\sigma_{0}\right.$, $\Delta \sigma_{\mathrm{lph}}$ и $\left.\sigma=\sigma_{0}+\Delta \sigma_{1 \mathrm{ph}}\right)$ для основного состояния $(1 s)^{2} 2 s \mathrm{Li}-$ подобных ионов. Для $A_{\text {fns }}$ и $B(\alpha Z) / Z$ для сравнения приведены результаты наших расчетов по теории возмущений и данные из работы [23]. Также представлены результаты для $\sigma_{0}=\alpha^{2} Z S(\alpha Z) / 12$ и $\Delta \sigma_{1 \mathrm{ph}}=\alpha^{2} B_{\mu}(\alpha Z) / 12$ из работы [18]. Значения $\sigma$ даны в единицах $10^{-3}$

\begin{tabular}{r|l|l|l|l|l}
\hline$Z$ & $A_{\text {fns }}$ & $B / Z$ & $\sigma_{0} \cdot 10^{3}$ & $\Delta \sigma_{1 \mathrm{ph}} \cdot 10^{3}$ & $\sigma \cdot 10^{3}$ \\
\hline 32 & 1.1196 & -0.0940 & 0.155542 & -0.008312 & 0.147230 \\
& $1.1196^{a}$ & $-0.0941^{a}$ & $0.155586^{b}$ & $-0.008325^{b}$ & 0.147261 \\
\hline 54 & 1.4062 & -0.0718 & 0.318991 & -0.011416 & 0.307574 \\
& $1.4061^{a}$ & $-0.0718^{a}$ & $0.319111^{b}$ & $-0.011416^{b}$ & 0.307695 \\
\hline 82 & 2.3958 & -0.0858 & 0.80409 & -0.02303 & 0.78106 \\
& $2.3973^{a}$ & $-0.0859^{a}$ & $0.80556^{b}$ & $-0.02303^{b}$ & 0.78254 \\
& $2.3987^{c}$ & $-0.0856^{c}$ & & & \\
\hline 83 & 2.4579 & -0.0872 & 0.83577 & -0.02377 & 0.8120 \\
& $2.4595^{a}$ & $-0.0873^{a}$ & $0.83650^{b}$ & $-0.02379^{b}$ & 0.8127 \\
& $2.4609^{c}$ & $-0.0870^{c}$ & & & \\
\hline 90 & 2.9795 & -0.0998 & 1.1013 & -0.03031 & 1.0710 \\
& $2.9803^{a}$ & $-0.0998^{a}$ & $1.1040^{b}$ & $-0.03036^{b}$ & 1.0736 \\
\hline 92 & 3.1613 & -0.1044 & 1.1960 & -0.03262 & 1.1633 \\
& $3.1641^{a}$ & $-0.1044^{a}$ & $1.1983^{b}$ & $-0.03263^{b}$ & 1.1657
\end{tabular}

Примечание. ${ }^{a}$ Теория возмущений, ${ }^{b}$ Moskovkin et al. [18], ${ }^{c}$ Shabaev et al. [23]

по теории возмущений и со значениями из работы [22] для $Z=82$ и 83.

В работе [18] было рассмотрено основное состояние $(1 s)^{2} 2 s$ Li-подобных ионов и вычислена поправка на однофотонный обмен. Мы сравниваем наши результаты для $\sigma_{0}$ и $\Delta \sigma_{1 \mathrm{ph}}$ с результатами работы [18] в табл. 2. В этом случае используются следующие соотношения: $\sigma_{0}=\alpha^{2} Z S(\alpha Z) / 12$ и $\Delta \sigma_{1 \mathrm{ph}}=\alpha^{2} B_{\mu}(\alpha Z) / 12$. Суммарное значение $\sigma=\sigma_{0}+\Delta \sigma_{1 \mathrm{ph}}$ также представлено в последней колонке. Результаты находятся в хорошем согласии, что может рассматриваться как подтверждение правильности использованных методов. Также представлены функции сверхтонкого расщепления $A_{\mathrm{fns}}$ и $B / Z$, результаты метода конечного поля сравниваются с теорией возмущений и со значениями, полученными из работы [23] для $Z=82$ и 83 .

В табл. 3 представлены наши результаты для основного состояния $(1 s)^{2}(2 s)^{2} 2 p_{1 / 2}$ В-подобных ионов. Значения $\sigma_{0}$ сравниваются с данными, представленными в работе [21]. Поправка на однофотонный обмен вычисляется в настоящей работе впервые. Мы также сравниваем значения $A_{\text {fns }}$ и $B / Z$, полученные в рамках метода конечного поля и теории возмущений. Для свинца и висмута также представлены результаты из работы [25]. Следует отметить, что результаты для Вподобных ионов демонстрируют две специфические тенденции. Во-первых, в отличие от состояний $1 s$ и $2 s$, где $\sigma$ быстро растет с $Z$, для состояния $2 p_{1 / 2} \sigma$ демонстри- 
Таблица 3. Коэффициенты сверхтонкого расщепления $\left(A_{\mathrm{fns}}\right.$ и $B / Z)$ и константа ядерного магнитного экранирования $\left(\sigma_{0}, \Delta \sigma_{1 \mathrm{ph}}\right.$ и $\left.\sigma=\sigma_{0}+\Delta \sigma_{1 \mathrm{ph}}\right)$ для основного состояния $(1 s)^{2}(2 s)^{2} 2 p_{1 / 2}$ В-подобных ионов. Для $A_{\text {fns }}$ и $B / Z$ для сравнения приведены результаты наших вычислений по теории возмущений и данные из работы [25]. Также представлены результаты для $\sigma_{0}$ из [21]. Значения $\sigma$ даны в единицах $10^{-3}$

\begin{tabular}{c|c|c|c|c|c}
\hline$Z$ & $A_{\mathrm{fns}}$ & $B / Z$ & $\sigma_{0} \cdot 10^{3}$ & $\Delta \sigma_{1 \mathrm{ph}} \cdot 10^{3}$ & $\sigma \cdot 10^{3}$ \\
\hline 32 & 1.11652 & -0.20966 & 4.7447 & 0.3522 & 5.0969 \\
& $1.11652^{a}$ & $-0.20949^{a}$ & $4.745^{b}$ & & \\
\hline 54 & 1.39693 & -0.17115 & 2.9612 & 0.1152 & 3.0764 \\
& $1.39693^{a}$ & $-0.17096^{a}$ & & & \\
\hline 82 & 2.43299 & -0.24117 & 2.2610 & 0.0226 & 2.2836 \\
& $2.43338^{a}$ & $-0.24085^{a}$ & $2.260^{b}$ & & \\
& $2.43344^{c}$ & $-0.23799^{c}$ & & & \\
\hline 83 & 2.50186 & -0.24729 & 2.2513 & 0.0243 & 2.2756 \\
& $2.50229^{a}$ & $-0.24697^{a}$ & $2.252^{b}$ & & \\
\hline 90 & $2.50229^{c}$ & $-0.24614^{c}$ & & & \\
\hline \multirow{2}{*}{92} & 3.1050 & -0.3032 & 2.2319 & 0.0018 & 2.2337 \\
& $3.1052^{a}$ & $-0.3028^{a}$ & & & \\
\hline \multirow{2}{*}{3.3262} & -0.3246 & 2.2403 & -0.0024 & 2.2379 \\
& $3.3271^{a}$ & $-0.3241^{a}$ & $2.240^{b}$ & &
\end{tabular}

Примечание. ${ }^{a}$ теория возмущений, ${ }^{b}$ Volchkova et al. [21], ${ }^{c}$ Glazov et al. [25].

рует поведение порядка $1 / Z$. Аналогичная особенность наблюдается для нелинейного эффекта Зеемана в Вподобных ионах [33,39-42], она объясняется вкладом состояния $2 p_{3 / 2}$ с очень малой разностью энергий в формуле (5). Во-вторых, поправка на однофотонный обмен имеет тот же знак, что и $\sigma_{0}$, и растет еще быстрее в направлении малых $Z$. Таким образом, для лёгких Bподобных ионов эффект ядерного магнитного экранирования заметно усиливается, а вклад межэлектронного взаимодействия значителен, и его следует учитывать во всех порядках теории возмущений.

\section{4. Заключение}

Константа ядерного магнитного экранирования вычислена для основного состояния $\mathrm{H}$-, $\mathrm{Li}^{-}$и В-подобных ионов в диапазоне $Z=32-92$ в рамках полностью релятивистского подхода. Для Li- и В-подобных ионов межэлектронное взаимодействие учтено в первом порядке теории возмущений. Использован метод конечного поля, основанный на решении уравнения Дирака с учетом магнитного и сверхтонкого взаимодействий. Полученные результаты сравниваются с ранее опубликованными значениями для H- и Li-подобных ионов. Для В-подобных ионов поправка на однофотонный обмен к константе ядерного магнитного экранирования получена впервые. Этот вклад становится особенно важен с уменьшением $Z$. Для ионов с малыми и средними $Z$ необходимы расчеты межэлектронного взаимодействия во всех порядках. Полученные результаты могут служить для определения ядерных магнитных моментов из высокоточных измерений $g$-фактора, ожидаемых в ближайшем будущем.

\section{Финансирование работы}

Исследование выполнено при финансовой поддержке РФФИ (гранты № 19-32-90278 и № 19-02-00974).

\section{Конфликт интересов}

Авторы заявляют, что у них нет конфликта интересов.

\section{Список литературы}

[1] Volotka A.V., Glazov D.A., Plunien G., Shabaev V.M. // Ann. Phys. (Berlin). 2013. V. 525. P. 636.

[2] Kozlov M.G., Safronova M.S., Crespo López-Urrutia J.R., Schmidt P.O. // Rev. Mod. Phys. 2018. V. 90. P. 045005.

[3] Shabaev V.M., Bondarev A.I., Glazov D.A., Kaygorodov M.Y., Kozhedub Y.S., Maltsev I.A., Malyshev A.V., Popov R.V., Tupitsyn I.I., Zubova N.A. // Hyperfine Interact. 2018. V. 239. № 60 .

[4] Indelicato P. // J. Phys. B. 2019. V. 52. P. 232001.

[5] Skripnikov L.V., Schmidt S., Ullmann J., Geppert C., Kraus F., Kresse B., Nörtershäuser W., Privalov A.F., Scheibe B., Shabaev V.M., Vogel M., Volotka A.V. // Phys. Rev. Lett. 2018. V. 120. P. 093001.

[6] Fella V., Skripnikov L.V., Nörtershäuser W., Buchner M.R., Deubner H.L., Kraus F., Privalov A.F., Shabaev V.M., Vogel M. // Phys. Rev. Research. 2020. V. 2. P. 013368.

[7] Werth G., Häffner H., Hermanspahn N., Kluge H.-J., Quint W., Verdú J., in: Karshenboim S.G. et al. (Eds.) // The Hydrogen Atom, Springer, Berlin. 2001. P. 204.

[8] Quint W., Moskovkhin D., Shabaev V.M., Vogel M. // Phys. Rev. A. 2008. V. 78. P. 032517.

[9] Sturm S., Vogel M., Köhler-Langes F., Quint W., Blaum K., Werth G. // Atoms. 2017. V. 5. P 4.

[10] Sturm S., Köhler F., Zatorski J., Wagner A., Harman Z., Werth G., Quint W., Keitel C.H., Blaum K. // Nature. 2014. V. 506. P. 467.

[11] Glazov D.A., Köhler-Langes F., Volotka A.V., Blaum K., Heiße F., Plunien G., Quint W., Rau S., Shabaev V.M., Sturm S., Werth G. // Phys. Rev. Lett. 2019. V. 123. P. 173001.

[12] Arapoglou I., Egl A., Höcker M., Sailer T., Tu B., Weigel A., Wolf R., Cakir H., Yerokhin V.A., Oreshkina N.S., Agababaev V.A., Volotka A.V., Zinenko D.V., Glazov D.A., Harman Z., Keitel C.H., Sturm S., Blaum K. // Phys. Rev. Lett. 2019. V. 122. P. 253001.

[13] Shabaev V.M., Glazov D.A., Plunien G., Volotka A.V. // J. Phys. Chem. Ref. Data. 2015. V. 44. P. 031205.

[14] Harman Z., Sikora B., Yerokhin V.A., Cakir H., Debierre V., Michel N., Oreshkina N.S., Belov N.A., Zatorski J., Keitel C.H. // J. Phys.: Conf. Ser. 2018. V. 1138. P. 012002.

[15] Moskovkin D.L., Oreshkina N.S., Shabaev V.M., Beier T., Plunien G., Quint W., Soff G. // Phys. Rev. A. 2004. V. 70. P. 032105.

[16] Yerokhin V.A., Pachucki K., Harman Z., Keitel C.H. // Phys. Rev. Lett. 2011. V. 107. P. 043004. 
[17] Yerokhin V.A., Pachucki K., Harman Z., Keitel C.H. // Phys. Rev. A. 2012. V. 85. P. 022512.

[18] Moskovkin D.L., Shabaev V.M., Quint W. // Opt. Spectrosc. 2008. V. 104. P. 637.

[19] Moskovkin D.L., Shabaev V.M. // Phys. Rev. A. 2006. V. 73. P. 052506.

[20] Moskovkin D.L., Shabaev V.M., Quint W. // Phys. Rev. A. 2008. V. 77. P. 063421.

[21] Volchkova A.M., Varentsova A.S., Zubova N.A., Agababaev V.A., Glazov D.A., Volotka A.V., Shabaev V.M., Plunien G. // Nucl. Instrum. Methods Phys. Res. B. 2017. V. 408. P. 89.

[22] Shabaev V.M., Tomaselli M., Kühl T., Artemyev A.N., Yerokhin V.A. // Phys. Rev. A. 1997. V. 56. P. 252.

[23] Shabaev V.M., Shabaeva M.B., Tupitsyn I.I., Yerokhin V.A., Artemyev A.N., Kühl T., Tomaselli M., Zherebtsov O.M. // Phys. Rev. A. 1998. V. 57. P. 149.

[24] Volotka A.V., Glazov D.A., Tupitsyn I.I., Oreshkina N.S., Plunien G., Shabaev V.M. // Phys. Rev. A. 2008. V. 78. P. 062507.

[25] Glazov D.A., Volotka A.V., Andreev O.V., Kosheleva V.P., Fritzsche S., Shabaev V.M., Plunien G., Stöhlker Th. // Phys. Rev. A. 2019. V. 99. P. 062503.

[26] Moore E.A. // Mol. Phys. 1999. V. 97. P. 375.

[27] Pyper N.C. // Mol. Phys. 1999. V. 97. P. 381.

[28] Pyper N.C., Zhang Z.C. // Mol. Phys. 1999. V. 97. P. 391.

[29] Ivanov V.G., Karshenboim S.G., Lee R.N. // Phys. Rev. A. 2009. V. 79. P. 012512.

[30] Rozenbaum E.B., Glazov D.A., Shabaev V.M., Sosnova K.E., Telnov D.A. // Phys. Rev. A. 2014. V. 89. P. 012514.

[31] Shabaev V.M., Tupitsyn I.I., Yerokhin V.A., Plunien G., Soff G. // Phys. Rev. Lett. 2004. V. 93. P. 130405.

[32] Sapirstein J., Johnson W.R. // J. Phys. B. 1996. V. 29. P. 5213.

[33] Varentsova A.S., Agababaev V.A., Volchkova A.M., Glazov D.A., Volotka A.V., Shabaev V.M., Plunien G. // Nucl. Instum. Methods Phys. Res. B. 2017. V. 408. P. 80.

[34] Volchkova A.M., Agababaev V.A., Glazov D.A., Volotka A.V., Fritzsche S., Shabaev V.M., Plunien G. // arXiv:2009.00109.

[35] Maltsev I.A., Shabaev V.M., Popov R.V., Kozhedub Y.S., Plunien G., Ma X., Stöhlker Th. // Phys. Rev. A. 2018. V. 98. P. 062709.

[36] Kotov A.A., Glazov D.A., Malyshev A.V., Vladimirova A.V., Shabaev V.M., Plunien G. // X-Ray Spectrom. 2020. V. 49. P. 110.

[37] Maltsev I.A., Shabaev V.M., Zaytsev V.A., Popov R.V., Kozhedub Y.S., Tumakov D.A. // Opt. Spectrosc. 2020. V. 128. P. 1100.

[38] Kotov A.A., Glazov D.A., Shabaev V.M., Plunien G. // Atoms. 2021. V. 9. P. 44.

[39] von Lindenfels D., Wiesel M., Glazov D.A., Volotka A.V., Sokolov M.M., Shabaev V.M., Plunien G., Quint W., Birkl G., Martin A., Vogel M. // Phys. Rev. A. 2013. V. 87. P. 023412.

[40] Glazov D.A., Volotka A.V., Schepetnov A.A., Sokolov M.M., Shabaev V.M., Tupitsyn I.I., Plunien G. // Phys. Scr. 2013. V. T156. P. 014014.

[41] Agababaev V.A., Volchkova A.M., Varentsova A.S., Glazov D.A., Volotka A.V., Shabaev V.M., Plunien G. // Nucl. Instrum. Methods Phys. Res. B. 2017. V. 408. P. 70.

[42] Varentsova A.S., Agababaev V.A., Glazov D.A., Volchkova A.M., Volotka A.V., Shabaev V.M., Plunien G. // Phys. Rev. A. 2018. V. 97. P. 043402. 